\title{
Review: diagnostic tests for bacterial rhinosinusitis have moderate accuracy; antibiotics reduce clinical failures
}

Lau J, Zucker D, Engels EA, et al. Diagnosis and treatment of acute bacterial rhinosinusitis. Rockville, MD: Agency for Health

Care Policy and Research; March 1999. AHCPR publication no. 99-E016. http://www.ahcpr.gov/clinic/sinussum.htm.

QUESTION: In symptomatic patients in primary care settings, what are the most

effective diagnostic tests, antibiotics, and ancillary treatments for bacterial rhinosinusitis

(paranasal sinus bacterial infection with symptoms for $\leqslant 4$ wks)?

\section{Data sources}

Studies were identified by searching Medline (1966 to May 1998) with the terms sinusitis and sinus with upper respir: and infect: (using various endings) and EMBASE/Excerpta Medica; scanning lists of conference abstracts and bibliographies of relevant articles and studies; and contacting experts.

\section{Study selection}

All studies included adults or children who had had symptoms for $\leqslant 4$ weeks. Diagnostic studies were selected if they compared the results of $\geq 2$ tests, including clinical criteria, radiographs, ultrasonography, or sinus puncture. Randomised controlled trials were selected if they compared antibiotics with placebo or compared $\geq 2$ antibiotics.

\section{Data extraction}

Patient, disease, and study characteristics; tests and treatments being compared; outcomes; and adverse effects.

\section{Main results}

14 studies of diagnostic tests, 74 of antibiotics, and 10 of ancillary treatments (decongestants, steroids, antihistamines, and proteolytic and mucolytic agents) met the inclusion criteria. All diagnostic tests had only moderate sensitivity and specificity (table 1). Any antibiotic had fewer clinical failures than no antibiotic treatment; comparison of specific antibiotics or classes of antibiotics showed no differences in clinical failures (amoxicillin or folate inhibitors $v$ other newer, more expensive antibiotics) (table 2). Ancillary treatments of rhinosinusitis have mixed results and are too dissimilar for pooling of data.

\section{Conclusions}

Clinical and radiological tests to diagnose acute bacterial rhinosinusitis have moderate sensitivity and specificity. Treatment with antibiotics has a lower clinical failure rate than does placebo treatment. Comparisons of individual antibiotics or classes of antibiotics show no differences.

\section{COMMENTARY}

The abundance of patients with rhinosinusitis symptoms and the problem of antibiotic overuse render this long (249 pages) summary and meta-analysis important. The report's thorough summary of the literature will be helpful to educators and investigators.

Any summary is limited by the quality of data available in the literature. The prevalence of acute bacterial rhinosinusitis in unselected symptomatic outpatients is important to decision making. However, the patient selection process is poorly described in many studies, and prevalence values have ranged between $15 \%$ and $85 \%$. The performance of clinical criteria in the diagnosis of bacterial infection is also quite important. Only 1 study (which was otherwise flawed) compared clinical criteria with sinus aspiration, the diagnostic standard. 3 other studies compared clinical criteria with radiographic diagnosis, which performs imperfectly (table 1).

Despite these limitations, a number of lessons important to primary care physicians are in this report. For diagnosis, the use of clinical criteria to guide treatment is usually the best strategy. The use of radiography or other imaging in the initial diagnostic evaluation is always much more expensive and never much more effective than other available strategies. A decision analysis included in this report showed that in most settings practitioners should use a clinical prediction rule to decide which patients to treat with amoxicillin or folate inhibitors. However, with mild symptoms or in low prevalence settings, a period of initial symptomatic treatment is reasonable. For initial antibiotic choice, the therapeutic equivalence of less expensive and more expensive antibiotics was maintained even in studies published after 1993. The choice of an expensive antibiotic as initial treatment is therefore not indicated, unless antibiotic allergies are present. Finally, although the data on ancillary treatments are sparse, 2 studies suggest that intranasal steroids may provide benefit when added to antibiotics.

Laura R Willett, MD Robert Wood Johnson Medical School New Brunswick, New Jersey, USA

Table 1 Diagnostic tests for acute bacterial rhinosinusitis*

\begin{tabular}{llllll} 
Comparison & $\begin{array}{l}\text { Number of } \\
\text { trials }\end{array}$ & $\begin{array}{l}\text { Sensitivity (95\% } \\
\text { CI) }\end{array}$ & Specificity (CI) & +LR & -LR \\
Sinus radiography $v$ puncture & & & & & \\
\hline$\quad$ Fluid or opacity definition & 5 & $73 \%(60$ to 83$)$ & $80 \%(71$ to 87$)$ & 3.7 & 0.3 \\
\hline$\quad$ Fluid, opacity, or mucosal thickening & 3 & $90 \%(68$ to 97$)$ & $61 \%(20$ to 91$)$ & 2.9 & 0.2 \\
\hline Clinical $v$ radiography & 3 & $57 \%(37$ to 74$)$ & $76 \%(60$ to 87$)$ & 2.4 & 0.6 \\
\hline
\end{tabular}

Table 2 Clinical failure rates with antibiotics for acute bacterial rhinosinusitis (treatment range 5 days to 7 weeks)*

\begin{tabular}{|c|c|c|c|c|}
\hline Comparison & $\begin{array}{l}\text { Number of } \\
\text { trials }\end{array}$ & $\begin{array}{l}\text { Weighted event } \\
\text { rates }\end{array}$ & RRR $(95 \%$ Cl) & NNT (Cl) \\
\hline Any $v$ no antibiotic & 6 & $16 \% \vee 31 \%$ & $46 \%(21$ to 63$)$ & 7 (4 to 25$)$ \\
\hline Folate inhibitors $v$ other antibiotics & 9 & $13 \% \vee 11 \%$ & $1 \%(-97$ to 48$)$ & Not significant \\
\hline
\end{tabular}

Source of funding: Agency for Health Care Policy and Research.

For correspondence: Dr J Lau, New England Medical Center, Division of Clinical Care Research, 750 Washington Street, Box 63, Boston, $M A$ 02111, USA.Fax +1 6176368023. 